\title{
PRÁT ICAS EDUCATIVAS EM DIABETES MELLITUS: revisão integrativa da literatura
}

Anna Karla de Oliveira T ito BORBA a , A na Paula de Oliveira M ARQUES M árcia Carréra Campos LEAL'c, Roberta Souza Pereira da Silva RAM OS ${ }^{d}$

\section{RESUMO}

T rata-se de uma revisão integ rativa da literatura com o objetivo de identificar a produção científica mul tiprofissional sobre as práticas educativas para indivíduos com diabetes, disponíveis nas bases de dados: Literatura LatinoA mericana em Ciências da Saúde (LIL ACS), M edical L iterature end R etrieval System O nline (M edline), ÍndiceB Bibliográfico E spañol en Ciencias de la Salud (I becs) e a Base de Dados em E nfermagem (BDE N F), no período de 1999 a 2009. Os resultados mostram que as práticas educativas são desenvolvidas, principalmente, para adultos e idosos de até 80 anos, e envolvem temáticas que refletem o cotidiano do viver com diabetes. Estas práticas são difundidas, principalmente através de grupos, trazendo benefícios, não só para 0 indivíduo com diabetes, mas também para 0 profissional de saúde. Visualiza-se o processo de mudança do paradigma da educação tradicional, para uma educação problematizadora e dialógica, com vistas à promoção da saúde.

D escritores: E ducação em saúde. Promoção da saúde. Diabetes M ellitus.

\section{RESUMEN}

Setrata deuna revisión integradora de la literatura con el fin deidentificar la producción científica multi-disciplinaria sobre la práctica educativa para los individuos con diabetes, di sponibles en bases dedatos: $L$ iteratura $L$ atino A mericana en Ciencias dela salud (L IL ACS), M edical L iteratureend R etrieval System on L ine(M edline), Índice Bi ibliográfico E spañol en Ciencias de la Salud (I becs) y L a Base de D atos de E nfermería (BDE N F ), durante el período entre 1999 y 2009. L os resultados muestran que las prácticas educativas se desar rollan, principalmente para adultos y personas mayores hasta los 80 años e involucran temas que reflejan la vida cotidiana de vivir con diabetes. E stas prácticas están muy extendidas, especialmente a través de grupos, con beneficios no solamente para el individuo con diabetes, sino también para el profesional de la salud. Visualizar el proceso de cambio del paradigma de la educación tradicional a una educación basada en problemas y el diálogo con miras a la promoción de la salud.

D escriptores: E ducación en salud. P romoción de la salud. D iabetes M ellitus.

T ítulo: $L$ as prácticas educativas en la diabetes M ellitus: revisión integradora de la literatura.

\section{ABST RACT}

This is an integrative literature review which aims to identify the multi-professional scientific production on educational practices for individuals with diabetes available in the databases: $\mathrm{L}$ atin A merican $\mathrm{L}$ iterature in $\mathrm{H}$ ealth $\mathrm{S}$ ciences ( $\mathrm{L}$ iteratura L atino A mericana em Ciências da Saúde - L I L ACS), M edical L iterature and R etrieval System online (M edline), Spanish B ibliographical Index in H ealth Sciences (ÍndiceB B ibliográfico E spañol en Ciencias de la Salud - I becs) and the D atabase on $N$ ursing (B ase de Dados em Enfermagem - BDEN F), from 1999 to 2009. Results show that educational practices are developed mainly for adults and seniors up to 80 years of age, and involve themes that reflect the daily life of living with diabetes. T hese practices are spread mainl y through groups, bringing benefits not onl y for the individual with diabetes but also for the healthcare professional. Thus, we can see the process of changing the traditional education paradigm to a problembased, dialogical education, with a view for promoting health.

Descriptors: $\mathrm{H}$ ealth education. Promotion of health. D iabetes M ellitus.

T itle: E ducational practices for diabetes M ellitus: integrativeliterature review.

\footnotetext{
a Enfer meira E specialista em Saúde da F amília, M estranda pelo Programa de Pós-G raduação em E nfermagem da U niversidade F ederal de Pernambuco (UFPE), Recife, Pernambuco, Brasil.

b D outora em Nutrição, D ocente do Programa de Pós-G raduação em Enfermagem da U F PE, Recife, Pernambuco, Brasil.

' D outora em Odontologia Preventiva e Social, D ocente do Programa de Pós-G raduação em Enfermagem da UF PE, Recife, Pernambuco, Brasil.

d Enfermeira Especialista em Saúde Pública, M estranda do Programa de Pós-Graduação em Enfermagem da U F PE, Recife, Pernambuco, Brasil.
} 


\section{INT RODUÇÃO}

A Educação Sanitária surge no Brasil no começo do século $X X$, para combater as epidemias de doenças infecto-contagiosas através de campanhas com uma abordagem biologista e mecanicista ${ }^{(1)}$. Na década de 70 , com o Relatório L al onde (1974) e a Conferência de A Ima-A ta (1978), os deter minantes sociais e econômicos são incluídos no processo saúde-doença, havendo a priorização das medidas preventivas e educativas direcionadas às mudanças comportamentais e de estilo de vida(2). A Promoção da Saúde é viabilizada pela educação em saúde, processo político de formação para a cidadania ativa, para a ação transformadora da realidade social, e, principalmente, na busca da melhoria na qualidade de vida ${ }^{(3)}$. A prática educativa pautada no diálogo e na troca de saberes valoriza 0 conhecimento popular, o estímulo e o respeito à autonomia do sujeito no cuidado de sua própria saúde, e o incentivo à participação ativa no controle social, com vistas a contribuir na melhoria das condições de vida e de saúde da população(4).

A educação em saúde é fundamental para as intervenções preventivas no âmbito comunitário, particularmente no que se refere às doenças crônicas. Entre elas, destaca-se o diabetes M ellitus, que conforme as estimativas da Organização M undial de Saúde (OM S), no ano de 2000, atingia cerca de 177 milhões de portadores em todo 0 mundo. A expectativa é de que em $2025^{(5)}$, este número chegue a 350 milhões de pessoas. A prática educativa, como parte integrante do cuidado de E nfermagem, objetiva à construção de um saber compartilhado sobre o processo saúde-doença-cuidado. A través da elaboração e do fortalecimento da cumplicidade, a educação assegura aos indivíduos, o direito de decidir quais estratégias são mais apropriadas para promover, manter e recuperar sua saú$d e^{(6)}$.

O diabetes $M$ ellitus, quando não mantido os níveis glicêmicos próximos à nor mal idade, podelevar a complicações altamente incapacitantes para a realização das atividades diárias e produtivas dos indivíduos ${ }^{(5)}$. D iante disto, têm-se buscado metodologias que favoreçam uma visão real do problema, principalmente no que diz respeito aos fatores relacionados às práticas de autocuidado do indivíduo no contex to familiar e comunitário, para que se possa prestar o cuidado adequado, de acordo com as suas necessidades.
A Prática Baseada em Evidências é um meio utilizado na busca de conhecimentos para oferecer uma melhor assistência ao indivíduo. Tem como propósito, encorajar a utilização de resultados de pesquisas na assistência à saúde prestada nos diversos níveis de atenção, reforçando a importância da análise para a prática clínica(7). A través desta metodologia, o enfermeiro pode planejar práticas educativas, de acordo com as reais necessidades do indivíduo com diabetes, o que contribui para a meIhoria de sua assistência.

$\mathrm{N}$ a tentativa de contribuir para a ampliação do conhecimento das ações na saúde, este estudo buscou identificar a produção científica multiprofissional no período de 1999 a 2009, sobre as práticas educativas para indivíduos com diabetes.

A relevância deste trabalho está em constituir uma oportunidade para a identificação das práticas educativas, até então desenvolvidas aos indivíduos com diabetes no Brasil, refletindo a nossa realidade nos diversos níveis de atenção à saúde, e um pensar crítico da atuação do profissional de saúde, frente a esta clientela.

\section{MÉT ODO}

T rata-se de uma revisão integrativa da literatura, considerada um método de estudo que possibilita a síntese do conhecimento de um determinado assunto, al ém de apontar lacunas, que precisam ser preenchidas com a realização de novos estu$\operatorname{dos}^{(8)}$. Para o desenvolvimento da presente revisão foram percorridas as seguintes etapas: estabelecimento da questão norteadora; seleção dos artigos e critérios de inclusão; extração dos artigos incluídos na revisão; aval iação dos estudos incluídos; interpretações dos resultados, e apresentação da revisão integrativa ${ }^{(9)}$.

Para orientar a pesquisa, el aborou-se a seguinte pergunta: Como estão sendo desenvolvidas as práticas educativas para os indivíduos com diabetes?

A coleta de dados ocorreu durante o mês de julho de 2010 e foram utilizadas na sel eção dos artigos, as seguintes bases de dados eletrônicos: Literatura L atino-A mericana em Ciências da Saúde (LILACS), M edical Literature and Retrieval System Online (M edline), Índice Bibliográfico E spañol en Ciencias de la Salud (I becs) e a Base de Dados em E nfermagem (BDENF). Empregaram-se os Descritores em Ciências da Saúde (D eCS): educação 
em saúde e diabetes M ellitus, resultando no total de 350 artigos.

Ainda nesta etapa, foi realizada leitura criteriosa dos títulos e resumos a fim de verificar a adequação aos seguintes critérios de inclusão: texto completo disponível online, no período de 1999 a 2009, dos quais, os resumos descrevessem práticas de educativas desenvol vidas para os indivíduos com diabetes, e cujos dados fossem col etados no Brasil, objetivando uma anál ise ajustada à nossa realidade. D escartaram-se artigos relacionados à educação formal técnica; artigos bibliográficos; estudos de val idação de instrumentos/ escalas; artigos que avaliavam apenas o conhecimento dos indivíduos com diabetes e teses. Ao final, foram pré-selecionados 13 artigos, lidos na íntegra.

Para avaliar a qualidade dos estudos selecionados foram utilizados dois instrumentos: o primeiro, adaptado do Critical A pppraisal Skills P rogramme (CASP) ${ }^{(10)}$ - Programa de habilidades em leitura crítica, integrante do "P ublic $\mathrm{H}$ ealth $\mathrm{R}$ esource U nit" (PHRU). 0 instrumento é composto por 10 itens (máximo 10 pontos), abrangendo: 1) objetivo; 2) adequação metodológica; 3) apresentação dos procedimentos teóricos e metodológicos; 4) seleção da amostra; 5) procedimento para a coleta de dados; 6) relação entre o pesquisador e pesquisados; 7) consideração dos aspectos éticos; 8) procedimento para a análise dos dados; 9) apresentação dos resultados; 10) importância da pesquisa. Os estu- dos foram classificados de acordo com as seguintes pontuações: 06 a 10 pontos (boa qual idade metodológica e viés reduzido), e mínima de 5 pontos (qualidade metodológica satisfatória, porém com risco de viés aumentado). N este estudo, optou-se por utilizar apenas os artigos classificados de 6 a 10 pontos.

0 segundo instrumento utilizado correspondeu à Classificação H ierárquica das $\mathrm{E}$ vidências para Avaliação dos Estudos(11), e contempla os seguintes níveis: 1) revisão sistemática ou metánalise; 2) ensaios clínicos randomizados; 3) ensaio clínico sem randomização; 4) estudos de coorte e de caso-controle; 5) revisão sistemática de estudos descritivos e qualitativos; 6) único estudo descritivo ou qualitativo; 7) opinião de autoridades e/ ou relatório de comitês de especialidades. Ao final da análise destes dois instrumentos, totalizaram-se oito artigos.

Os artigos foram descritos de acordo com 0 público-al vo, local de real ização das práticas, embasamento teórico, recurso pedagógico, e identificação dos benefícios das práticas educativas para o indivíduo e para o profissional de saúde.

\section{RESULT ADOS E DISCUSSÃO}

$\mathrm{Na}$ T abela 1, verificam-se os resultados encontrados a partir da busca pelos descritores, conforme cada base de dados.

T abela 1 - Distribuição dos artigos encontrados e selecionados por bases de dados. Recife, PE , 2010.

\begin{tabular}{lcccc}
\hline \multirow{2}{*}{ Base de dados } & \multicolumn{4}{c}{ Artigos } \\
\cline { 2 - 5 } & Encontrados & Pré-selecionados & Excluídos & Analisados \\
\hline LILACS & 96 & 20 & 14 & 6 \\
IBECS & 8 & 1 & 1 & - \\
M edline & 182 & 9 & 7 & 2 \\
BDEN F & 64 & 11 & 11 & - \\
\hline Total & $\mathbf{3 5 0}$ & $\mathbf{4 1}$ & $\mathbf{3 3}$ & $\mathbf{8}$ \\
\hline
\end{tabular}

A base de dados que recuperou o maior número de publicações foi a M edline (182), seguida pela LILACS (96), BDENF (64) eIBECS (8). A pesar de a $M$ edline recuperar o maior número de estudos, a base LILACS foi a que obteve o maior número de estudos incluídos.

0 idioma português foi predominante $(87,5 \%)$, correspondendo a sete publicações. E ste resultado traduz a incipiência de pesquisas desenvolvidas no
Brasil, e publicadas em periódicos estrangeiros, assim como, pode estar associado ao fato de que a maioria dos estudos, seja oriunda da base de dados LILACS, que abrange estudos da A mérica Latina.

U m aspecto da estratégia de busca, que levou à exclusão de vários artigos em etapa posterior, diz respeito ao emprego do descritor educação em saúde. No entanto, a leitura do artigo na íntegra, 
revelava que os mesmos, não descreviam o desenvolvimento ou avaliação de práticas educativas. A lém disso, alguns artigos selecionados, ainda constavam em mais de uma base de dados: LI LACS (3), M edline (3), BDENF (4), considerado critério de exclusão.

E $m$ relação ao tipo de revista, dois artigos foram publicados em revistas de enfermagem geral, um em revista médica, e cinco em revistas de outras áreas da saúde. Acredita-se que a maior frequência de publicações em periódicos nas diversas áreas da saúde, comprometa a atual ização desse conhecimento pela ár ea de enfermagem.

Quanto à autoria dos artigos incluídos, quatro são de enfermeiros, um tem entre seus autores enfermeiros e médico, um foi redigido por enfermeiro, médico e psicólogo, um possui apenas fisioterapeuta, e outro foi escrito por fonoaudiólogo, médico e assistente social.

A presença do enfermeiro como facilitador do processo educativo foi identificada, em seis, entre os oito estudos encontrados. A educação em saúde está inserida no contexto da atuação da en- fermagem, como meio para o estabelecimento de uma relação dialógico-reflexiva entre enfer meiro e cliente, devendo este, conscientizar-se sobre sua situação de saúde-doença, e perceber-se como sujeito de transformação de sua própria vida ${ }^{(12)}$. Quanto ao tipo de delineamento de pesquisa, evidenciou-se na amostra: cinco estudos descritivos ou qualitativos, e três estudos de coortes. Dessa forma, em relação à força das evidências ${ }^{(11)}$ obtidas nos artigos, encontrou-se três com nível IV, e cinco, com nível de evidência VI. A abordagem da pesquisa qualitativa e descritiva per mite responder às questões relacionadas à percepção dos indivíduos em relação à ação educativa, bem como, possibilita entender as melhores intervenções desenvolvidas, com vistas à promoção da saúde. E nos estudos de coorte é possível documentar se houve mudança no comportamento dos indivíduos após a participação no processo educativo. No Quadro 1, apresenta-se a caracterização das práticas educativas desenvolvidas para indivíduos com diabetes no Brasil, descrita nos artigos incluídos na revisão integ rativa(13-20).

\begin{tabular}{|c|c|}
\hline Variáveis & Caracterização das Práticas E ducativas \\
\hline Local & Comunidade ${ }^{(13,17)}$, Posto de Saúde ${ }^{(19,20)}$, A mbulatório ${ }^{(14,16)}$ e H ospital ${ }^{(15)}$ \\
\hline Público-A lvo & $\begin{array}{l}\text { A dolescente(19), A dulto }{ }^{(14-19)} \text {, I doso de até } 80 \text { anos }^{(14,15,16,18)} \text {, A cima de } 80 \text { anos }^{(19)} \text { e F amilia- } \\
\text { res }^{(14,18)}\end{array}$ \\
\hline $\begin{array}{l}\text { E mbasamento } \\
\text { T eórico }\end{array}$ & $\begin{array}{l}\text { Concepç̃oses de Paulo F reire }{ }^{(13,16,17)} \text {, T eoria do Autocuidado de D orothea Orem }{ }^{(16)} \text {, T eoria de } \\
\text { Piaget e Vygostky }\end{array}$ \\
\hline $\begin{array}{l}\text { Recurso } \\
\text { Pedagógico }\end{array}$ & $\begin{array}{l}\text { A ulas expositivas }{ }^{(19,20)} \text { e tel evisivas; atividades grupais (grupo focal }{ }^{(14,15)} \text {, grupo de convi- } \\
\text { vência } a^{(16)} \text {, grupo de ajuda mútua, círculos de discussão(13,18,20), jogos educativos }{ }^{(14)} \text {, livre as- } \\
\text { sociação de figuras e fotog rafias } \\
\text { atividades relacionar objetos com práticas com o uso de bonecos }{ }^{(17))}\end{array}$ \\
\hline Temas & 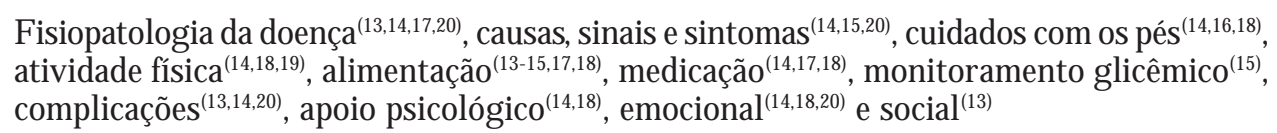 \\
\hline $\begin{array}{l}\text { Benefícios - } \\
\text { Indivíduo com } \\
\text { diabetes }\end{array}$ & M otivação $0^{(15,19)}$, independência ${ }^{(13,16)}$, autocuidado ${ }^{(14-18)}$ e controle metabólico ${ }^{(14,18-20)}$ \\
\hline $\begin{array}{l}\text { Benefícios - } \\
\text { Profissional }\end{array}$ & $\begin{array}{l}\text { Estabelecimento de laços de amizade }(14,16) \text {, apoi }{ }^{(16,20)} \text {, entrelaçamento entre a teoria e a } \\
\text { prática com uma visão holística do indivíduo } 0^{(13-15,17,18)}\end{array}$ \\
\hline
\end{tabular}

Q uadro 1 - Distribuição das variáveis e as respectivas características das práticas educativas. Recife, PE , 2010.

0 apoio educacional pode ter um impacto positivo sobre o comportamento das pessoas com diabetes, mediante mudanças no estilo de vida e consequente equilíbrio metabólico, refletindo no controle da evolução da doença e na redução dos custos de atendimento à saúde. 
A educação em saúde objetiva a melhoria das condições de vida e de saúde da população, e para isto, faz-se necessário que esteja voltada para a realidade a qual se destina, e contemple o espaço onde são vivenciados os principais problemas de saú$\mathrm{de}^{(21)}$. T al afirmativa corrobora com os achados deste estudo, em que todos os níveis de atenção à saúde foram cenários de práticas educativas no B rasil. D estaca-se que estas ações, envolvendo comunidades, serviços de saúde vinculados à atenção básica, escolas, creches, hospitais e outros locais, deve compreender as relações entre os sujeitos sociais, com uma abordagem ativa do sujeito ${ }^{(22)}$.

Ser viço de saúde que contemple saúde e educação, associado a procedimentos e embasamento teórico específicos, contrapõe-se a visão reducionista da execução do cuidado, e abre-se para uma nova realidade, com perspectiva de resgatar as práticas de saúde que, efetivamente, contribuam para a melhoria da assistência e da qualidade de vida das pessoas com diagnóstico de diabetes M ellitus ${ }^{(23)}$.

Quanto ao público-alvo das atividades educativas, as fases nas quais estão incluídos, adultos e idosos de até 80 anos (com média de idade de 76 anos) foram as mais apontadas dentro do ciclo de vida. A atenção para as questões de saúde no enveIhecimento tem crescido nas últimas décadas, principalmente no que diz respeito às doenças crônicas. Porém, nesta revisão foram insuficientes os achados direcionados para pessoas acima de 80 anos. Conforme dados do Censo D emográfico 2010, esta faixa etária vem aumentando em ritmo acelerado, reflexo do rápido envelhecimento populacional (24).

A promoção de práticas saudáveis deve ser objeto de ações educativas com os idosos, na busca da manutenção da saúde, autonomia na velhice e adaptação às modificações exigidas para o controle metabólico. No entanto, as ações educativas em saúde não deter minam diretamente a interferência nos determinantes sociais do envel hecimento ativo, mas podem oferecer contribuição significativa, ao expressar em vivamente o compromisso social do sistema de cuidados, e partilharem com os idosos os desafios nesta direção(25).

A participação da família nas práticas educativas para indivíduos com diabetes ainda é minoria, conforme os resultados deste estudo. A família representa uma unidade de cuidado influen- ciada pelo sistema de crenças, valores e significados compartilhados, que são fundamentais para que o indivíduo com diabetes consiga al cançar 0 automanejo da doença. 0 processo de tomada de decisão é mediado por esses fatores que permeiam a relação com os serviços de saúde e que determinam, na maioria das vezes, a utilização dos recursos de tratamento ${ }^{(26)}$

Ao considerar que a participação familiar no processo educativo contribui para a adesão à terapêutica, ao se torna permeável aos ajustamentos necessários ao autocuidado em diabetes, cabe ao profissional de saúde inserir a família na sua práxis diária e envolvê-la como parte integrante do seu cuidar, através da educação em saúde ${ }^{(27)}$. A compreensão do cuidado, em todas as etapas do ciclo de vida do indivíduo, pressupõe-se um referencial teórico e filosófico, assim como, a compreensão da experiência de cuidado no contexto sócio-político, econômico e cultural em que ocorre, busca a potencialização do indivíduo como cidadão.

Historicamente, a pauta que os profissionais de saúde vêm aplicando em sua prática educativa, segue abordagens que se assemel ham à metodologia pedagógica autoritária, reflexo do modeIo sanitarista. N esta abordagem, o educar em saúde acontece simplesmente com a transmissão de conhecimentos de forma verticalizada, do educador em relação ao educando $0^{(1,28)}$.

Contudo, identifica-se que três, entre os oito estudos trazem a abordagem construtivista da aprendizagem proposta por Paulo Freire. Segundo 0 autor, a educação se dá mediante a problematização das situações vividas, e implica em um retorno crítico a elas. Ela acontece no diálogo, onde a reflexão e a ação orientam-se para o mundo que precisa ser transformado; com a liberdade de criar, de propor, o quê e como aprender; e com a conscientização, que significa assumir uma posição crítica frente à real idade ${ }^{(4,17)}$.

$\mathrm{Na}$ educação dialógica pressupõe-se a compreensão do outro como sujeito, detentor de um determinado conhecimento, e não, mero receptor de informações. Isso implica no respeito ao univer so cultural dos participantes, e principal mente na ideia de saberes, popular e científico pensados de for ma dinâmica. E ntende-se que em um processo contínuo de interação, postura de "escuta atenta" e "abertura" ao saber do outro, dá-se a possibilidade de uma construção compartilhada do co- 
nhecimento e de formas de cuidado diferenciadas $^{(22) \text {. }}$

Tendo em vista a cronicidade do diabetes, a educação em saúde, no contexto da educação emancipatória, implica em capacitar os indivíduos com doença à problematizar sobre sua condição, desencorajando a acomodação e discutindo as opções, visando a mudança de uma realidade passível de ser mudada por el es ${ }^{(29)}$. A T eoria do Autocuidado de D orothea Orem também esteve presente entre os artigos selecionados. Esta abordagem se ajusta aos propósitos da educação em saúde, e se vislumbra à promoção da autonomia do sujeito, ao reconhecer a pessoa como aquela que tem direito de exercer o controle sobre si e sua assistência, considerando seus valores, crenças, nível de conhecimento, habilidades e motivação(30).

As bases teóricas norteiam o cuidado de enfermagem dando sustentação a uma prática humanizada. Ao assistir o indivíduo com diabetes, destaca-se a necessidade do ol har holístico, num entendimento profundo e significativo da pessoa com a doença, e da diversidade dos contextos socioculturais que estão inseridos neste processo.

E mbora o diabetes possua uma dimensão complexa e envolva aspectos biológicos, sociais, culturais, psicológicos e econômicos, a temática mais preval ente nos artigos avaliados diz respeito à alimentação. Os hábitos alimentares são construídos ao longo da vida e são influenciados pelo convívio social e familiar, exigindo estratégias de ação voltadas para a população, considerando os aspectos culturais apresentados por ela(31). Para a determinação das necessidades de saúde e de educação de uma população, faz-se necessário conhecer, observar, e questionar a realidade concreta, abrindo espaços participativos para os relatos das vivências, experiências, expectativas e necessidades do indivíduo com diabetes. D essa forma, a educação problematizadora proporciona ao educador e ao educando, a oportunidade de se tor narem agentes ativos, participantes da transfor mação social, possibilitando a ambos observar a realidade, refletir sobre ela e buscar os meios possíveis para modificá-la(4).

As estratégias didáticas que transformem indivíduos socialmente inseridos no mundo é um dos pilares da educação em saúde, na medida em que amplia a capacidade de compreensão dos determinantes do ser saudável. No entanto, neste es- tudo, identificam-se resquícios da educação tradicional em saúde, através de aulas expositivas e televisivas, porém as atividades grupais foram as mais apontadas entre os recursos pedagógicos, possibilitando ações efetivas de promoção e educação em saúde.

U m grupo não é apenas um somatório de pessoas, mas, uma nova entidade com leis e mecanismos próprios, podendo oferecer suporte, realizar tarefas, socializar, melhorar o autocuidado ou oferecer psicoter apia. E stes espaços favorecem a construção coletiva de conhecimento e a reflexão acerca da realidade vivenciada pelos seus membros, por meio da quebra da relação vertical (profissionalcliente), valorização dos diversos saberes e da possibilidade de intervir criativamente no processo de saúde-doença de cada pessoa ${ }^{(32)}$. A convivência com um grupo que congrega pessoas com problemas semelhantes, como é o caso do diabetes, ajuda os integrantes a quebrarem barreiras, especialmente pela possibilidade de receber em feedback e sugestões construtivas de outras pessoas que vivenciaram ou vivenciam os mesmos problemas $^{(33)}$.

As práticas educativas trazem como benefícios para o indivíduo com diabetes, além do controle metabólico, o suporte quanto aos aspectos psicológicos e relacionamento familiar. Já para o profissional de saúde, possibilita o entrelaçamento entre a teoria e a prática, com uma visão global do sujeito como portador de uma doença crônica, cidadão e ser humano.N a compreensão freiriana, 0 profissional de saúde, como facilitador do processo educativo, deve colaborar para a apreensão de conhecimentos novos e ressignificação dos já existentes, estimulando os participantes a encontrar estratégias coletivas de enfrentamento dos problemas vividos pelos indivíduos, a nível primário, secundário ou terciário de atenção a saúde ${ }^{(6,34)}$.

No entanto, faz-se necessário uma maior integração entre os membros da equipe prestadora de assistência ao indivíduo com diabetes, o querespeita os preceitos da promoção da saúde proposto pela Carta de Ottawa $a^{(35)}$. N ão basta apenas atuar em equipe multiprofissional, mas, principalmente, de forma interdisciplinar, reconhecendo o indivíduo como corpo e mente, indissociáveis, para que as ações sejam planejadas e desenvolvidas em conjunto, possibilitando o compartilhamento da responsabilidade terapêutica entre as várias categorias profissionais. 
U m instrumento que facilita o processo educativo é a comunicação com o cliente. A troca de saber es entre o científico e o senso comum através do diálogo participativo contribui para a formação do saber, aprender e ensinar. Além disso, apresenta-se como uma estratégia para reflexão e discussão das situações de saúde, levando à tomada de consciência, o que conduz a um melhor enfrentamento das situações vivenciadas.

\section{CONCLUSÕES}

As práticas de educação em saúde realizadas no Brasil pelos profissionais de saúde, descritas na literatura pesquisada, enfocam um processo de mudança do paradigma da educação bancária, reflexo do model o assistencial sanitarista, para o enfoque da educação problematizadora e dialógica com vistas à promoção da saúde.

A participação de todos os envolvidos nas práticas de saúde construtivistas mostra-se um método efetivo e traz muitos benefícios para o indivíduo com diabetes e o profissional, principalmente na construção do autocuidado consciente, e do controle metabólico, contribuindo para a melhoria da qualidade de vida. D estaca-se também, o papel do enfermeiro integ rado a equipe inter disciplinar, que tem na educação em saúde, o eixo norteador da sua assistência.

A realização desta pesquisa evidencia a necessidade da construção do conhecimento científico, com enfoque para as práticas educativas com idosos acima de 80 anos, grupo de risco para o desenvolvimento de doenças crônicas ${ }^{(36)}$, reflexo da transição demográfica. Além do segmento idoso, os familiares ainda são pouco envolvidos no processo educativo.

D entre as limitações identificadas nos estudos analisados, nota-se que, apesar das contribuições dadas pelas pesquisas que refletem a realidade brasileira sobre o tema em questão, há a necessidade da atuação em equipe interdisciplinar. Sugere-se então, que além do aprofundamento da temática para o levantamento dos possíveis entraves deste processo, possa-se também criar estratégias de capacitação dos profissionais de saúde que prestam assistência aos indivíduos com diabetes, sob o aspecto de uma educação crítica e transformadora, para contemplar as necessidades biopsicossociais em suas ações individuais e coletivas.

\section{REFERÊNCIAS}

1 M arjorie E D M . E ducação em saúde: conceitos e propósitos. Cogitare Enferm. 2009;14(4):773-6.

2 Arantes $R C$, M artins JLA, Lima M F, Rocha RM N, Silva RC, Villela W V. Processo saúde-doença e promoção da saúde: aspectos históricos e conceituais. Rev APS. 2008;11(2):189-98.

3 Pelicioni M CF, Pelicioni AF. E ducação e promoção da saúde: uma retrospectiva histórica. M undo Saúde. 2007;31(3):320-8.

4 F reire $P$. Pedagogia do oprimido. 50a ed. R io de Janeiro: Paz e Terra; 2011.

5 Sociedade Brasileira de D iabetes. T ratamento e acompanhamento do diabetes M el litus: diretrizes da Sociedade Brasileira de Diabetes [ Internet]. Rio de Janeiro; 2007 [ citado 2010 jun 20] . Disponível em: http:/ / www.diabetes.org.br/ educacao/ docs/ Diretrizes_ SBD_2007.pdf.

6 Budó $M L$, M attioni FC, Silva F M, Schimith M D. $\mathrm{H}$ ealth education towards the chronic disease bearer: implications with the social network. Ciênc Cuid Saúde. 2009;8(Supl):142-7.

7 M endes KDS, Silveira RCCP, G alvão CM . Revisão integrativa: método de pesquisa para a incorporação de evidências na saúde e na enfermagem. T exto Contexto Enferm. 2008;17(4):758-64.

8 Polit D F, Beck CT, H ungler BP. F undamentos de pesquisa em enfermagem: métodos, avaliação e utilização. 6a ed. Porto Alegre: Artmed; 2006.

9 Beya S, Nicoll LH. W riting an integrative review. AOR N J. 1998;67(4):877-80.

10 M ilton Keynes Primary CareT rust. Critical Appraisal Skills Programme. London: Oxford; 2002.

11 Stillwell S, M elnyk BM, F ineout-Overholt E, W illiamson K. E vidence-based practice: step by step. Am J N urs. 2010;110(5):41-7.

12 Sousa L B, T orres CA, Pinheiro P N C, Pinheiro AKB. Práticas de educação em saúde no Brasil: a atuação da enfermagem. Rev E nferm U ERJ. 2010;18(1):55-60.

13 Rêgo M A B, N akatani AY K, Bachion M M . E ducação para a saúde como estratégia de inter venção de enfermagem às pessoas portadoras de diabetes. Rev G aúcha E nferm. 2006;27(1):60-70.

14 Torres HC, Hortale VA, Schall V. A experiência de jogos em grupos operativos na educação em saúde para diabéticos. Cad Saúde Pública. 2003;19(4):1039-47. 
15 Comiotto G, M artins JJ. Promovendo o autocuidado ao indivíduo portador de diabetes: da hospital ização ao domicílio. A CM A rq Catarin M ed. 2006;35(3):5964.

16 Coelho M S, Silva DM GV. Grupo educação-apoio: visualizando o autocuidado com os pés de pessoas com diabetes mellitus. Ciênc Cuid Saúde. 2006; $5(1): 11-5$.

17 Vechi AP, Santos AF, Scatolin BE, Rodrigues IC, Oliveira M P, A raújo RR DF. U ma prática al ternativa de ensinar o portador de doença crônica. A rq Ciênc Saúde. 2007;14(2):113-7.

18 Selli L, Papaléo LK, M eneghel SN, Torneros J Z. T écnicas educacionales en el tratamiento de la diabetes. Cad Saúde Pública. 2005;21(5):1366-72.

19 Osawa F H , Caromano FA. Avaliação da adesão a um programa de atividade física por portadores de diabetes mellitus e/ ou hipertensão arterial. A rq Ciênc Saúde U nipar. 2002;6(3):127-30.

20 Silva TR, Feldman C, Lima M HA, Nobre M RC, Domingues RZL . Controle de diabetes mellitus e hipertensão arterial com grupos de intervenção educacional e ter apêutica em seguimento ambulatorial de uma unidade básica de saúde. Saúde Soc. 2006;15(3):180-9.

21 Lopes EM , Anjos SJ SB, Pinheiro AKB. Tendência das ações de educação em saúde realizadas por enfermeiros no Brasil. Rev Enferm UERJ. 2009;17 (2):273-7.

22 A cioli S. A prática educativa como expressão do cuidado em Saúde Pública. Rev Bras Enferm. 2008; 61(1):117-21.

23 G amba M A, G otlieb SLD, Bergamaschi D P, Vianna LAC. A mputações de extremidades inferiores por diabetes mellitus: estudo caso-controle. Rev Saúde Pública. 2004;38(3):399-404.

24 I nstituto Brasileiro de G eografia e E statística. Censo demográfico: Brasil 2010. Rio de Janeiro; 2011.

25 A ssis M . E nvelhecimento ativo e promoção da saúde: reflexão para as ações educativas com idosos. Rev APS. 2005;8(1):1-15.

\section{Endereço da autora / Dirección del autor / Author's address:}

Anna Karla de Oliveira T ito Borba

Rua Dr. José M aria, 900 , ap. 2401, Torre B, Rosarinho 52041-000, Recife, PE

E-mail: anninhatito@ hotmail.com
26 Zanetti M L, Biagg M V, Santos M A, Péres DS, T eixeira CRS. 0 cuidado à pessoa diabética e as repercussões na família. Rev Bras E nferm. 2008;61(2): 186-92.

27 Souza L M , W egner W, G orini M IPC. H ealth education: a strategy of care for the lay caregiver. Rev L atino-A m E nfermagem. 2007;15(2):337-43.

28 Penna CM M , Pinho L M O. A contramão dos programas de educação em saúde: estratégias de diabéticos. Rev Bras Enferm. 2002;55(1):7-12.

29 G rossi SAA. 0 manejo do diabetes mellitus sob a per spectiva da mudança comportamental . I n: G rossi SAA, Pascali P M , organizadoras. Cuidados de enfermagem em diabetes mellitus: manual de enfermagem. São Paulo: Sociedade Brasileir a de D iabetes; 2009. p. 18-31.

30 Orem D. Nursing concepts of practice. 5a ed. St. L ouis: M osby; 1995.

31 Costa JA, Balga RSM , Alfenas RCG, Cotta R M M Promoção da saúde e diabetes: discutindo a adesão e a motivação de indivíduos diabéticos participantes de programas de saúde. Ciênc Saúde Colet. 2011; 16(3):2001-9.

32 D ias VP, Silveira DT, W itt R R. E ducação em saúde: o trabalho de grupos em atenção primária. Rev APS. 2009;12(2):221-7.

33 F rancioni F F, Silva D G V. 0 processo de viver saudável de pessoas com diabetes mellitus através de um grupo de convivência. T exto Contexto E nferm. 2007; 16(1):105-11.

34 Souza AC, Colomé ICS, Costa L E D, Oliveira D L LC. A educação em saúde com grupos na comunidade: uma estratégia facilitadora da promoção da saúde. Rev G aúcha E nferm. 2005;26(2):147-53.

35 M inistério da Saúde (BR), Secretaria de Políticas de Saúde, P rojeto P romoção da Saúde. A s cartas de promoção da saúde [ I nter net] . Brasília (DF ); 2006 [ citado 2011 dez 20] . D isponível em: http:/ / bvsms. saude. gov.br/ bvs/ publicacoes/ cartas_promocao.pdf.

36 Veras R. E nvel hecimento populacional contemporâneo: demandas, desafios e inovações. Rev Saúde Pública. 2009;43(3):548-54.

Recebido em: 24/ 12/ 2010

A provado em: 01/ 03/ 2012 